\title{
Pre-cancer Lesions in the Cervix and Cervical Cancer Explained by Fooling of Homeostasis Hypothesis
}

\section{Pushpam Kumar Sinha*}

Independent Researcher, Vijayshree Complex, Kankarbagh, Patna, India

*Corresponding Author: Pushpam Kumar Sinha, Independent Researcher,

Vijayshree Complex, Kankarbagh, Patna, India.
Received: March 30, 2021

Published: April 09, 2021

(C) All rights are reserved by Pushpam Kumar Sinha.

\section{Abstract}

The three common disease states of the cervix upon having undergone Human Papillomavirus infection are Low-grade Squamous Intraepithelial Lesion, High-grade Squamous Intraepithelial Lesion, and Cervical Cancer. The genesis of all the three have been explained using the Fooling of Homeostasis Hypothesis. According to this hypothesis, for cancers that have stem cell basis, the would-be cancer cells just before turning cancerous undergo aggressive proliferation and aggressive apoptosis (at equal rate) thereby leading to fooling of the homeostasis maintained by the surrounding healthy cells and immune cells. This hypothesis predicts that the possible cure for Cervical Cancer could be the use of chemicals ethanol-alcohol dehydrogenase and sodium metabisulphite.

Keywords: Lesion; Cervix; Homeostasis; Cancer Stem Cells; Innate Immunity; Cell-mediated Immunity

\section{Introduction}

The three stages of the development of disease upon Human Papillomavirus (HPV) infection in the cervix of women are Lowgrade Squamous Intraepithelial Lesion (LSIL), High-grade Squamous Intraepithelial Lesion (HSIL), and Cervical Cancer (CC). All the three stages can be detected/diagnosed by regular and appropriate screening [1]. LSIL and HSIL are pre-cancer lesions. More than half of the women suffer HPV infection upon their first encounter with sex and show development of LSIL; $90 \%$ of LSILs go away naturally (without treatment) within 3 years [2]. Hence the infection associated with LSIL can be regarded as transient infection. Out of the rest $10 \%$ of LSILs that do not go away naturally, a very small fraction can transform into cancer, and the rest transforms to HSIL. The infection associated with HSIL can be regarded as persistent infection, which means that HSIL cannot go away without treatment. If left untreated, HSIL can transform to CC. The development of full-blown CC is a very slow process, taking 5 - 10 years after first exposure of the cells of cervix to HPV; several oth- er cancers have also been shown to be evolutionary, the evolution marked by multi-stage genetic mutations taking years [3]. Out of the women infected with HPV, merely less than $0.05 \%$ has a chance of developing CC [2]. Converted to absolute number, this mere percentage less than 0.05 transforms to nearly 570000 new CC cases per year, and this is a big number [4]. Hence there is significance of the study of CC. To understand CC and its treatment better, we must investigate the mechanism underlying both the pre-cancer lesion and the CC. I propose in this paper that this underlying mechanism is "Fooling of Homeostasis". Hence, before moving ahead let me first discuss the Fooling of Homeostasis Hypothesis (FHH) as proposed by me in [5].

At the heart of the FHH is Cancer Stem Cell (CSC). The widely accepted theory of carcinogenesis nowadays is that in many cancers the full-blown blast cells of the cancer are born out of and sustained by CSCs [5]. CSCs are formed from Adult Stem Cells (ASCs), or Progenitor Cells (PCs), or (in some cases, like, for e.g. liver cancer [6]) terminally differentiated cells by accumulating multiple genet- 
ic mutations spread over many years. But CSCs are alike ASCs in many respects; when cancer of any particular organ has developed CSCs maintain themselves in few numbers, are hardly undergoing proliferation, and have same self-renewing capability as ASCs. But there is a question that has largely remained unanswered for years, and that question is, "What exactly is the mechanism by which CSCs give birth to the blast cells of cancer?". FHH exactly answers this question. Immediately after formation, CSCs undergo proliferation at a high rate and at an equally high rate they also undergo apoptosis. The argument in support of the high apoptosis of proliferating CSCs is that internally the cells have a sense that they are defective in lieu of them being multiply mutated, thereby they program their own death by mechanisms internal to the cell. Because CSCs are undergoing apoptosis at a rate equal to that of their proliferation they fool the homeostasis, i.e., the normal healthy cells and the immune cells in the micro-environment of CSCs are unable to take any corrective action against or launch an attack on defective CSCs. The high apoptosis of CSCs is being interpreted (by cells themselves) as damage done to the cells. It is known from literature [7] that when the cells are undergoing damage the particular glucose metabolic pathway known as the pentose-phosphate pathway is up-regulated thereby leading to enhanced nucleic acid synthesis, enhanced proliferation, and reduced apoptosis. It is exactly this that happens to the CSCs, and thereby they escape homeostasis to establish cancer.

Natural regression of LSIL, the genesis of HSIL, and the genesis of CC explained

In the diseases of the cervix caused by HPV infection, the attack by the immune system has two targets: the virus itself, and the pre-cancer lesion or tumor. The border between the two types of attacks is very thin, i.e., there may be overlaps. Innate immunity (II) and Cell Mediated immunity (CMI) are the dominant players of immunity against the HPV infection of the cervix [2,4]. II is mostly the immune attack against the virus itself, whereas CMI is the one against the pre-cancer lesion or tumor. Humoral immunity (HI) as mediated by B cells unexpectedly has adverse effect on the lesions of the cervix [4]. Hence I do not discuss HI further, here.

Based on my description of $\mathrm{FHH}$, a possible explanation for natural regression of LSIL is the absence of "Fooling of Homeostasis" mechanism in the abnormal cells of the lesion. 21 strains of HPV have been identified as high risk as far as their role in progression of the diseases of the cervix towards CC is concerned [2]; out of these 21, the 2, HPV-16 and HPV-18 are the most prevalent ones. A marked feature of HPV infection, like any other viral infection, is the merger of the viral RNA with host DNA; and post-merger the expression of the viral proteins E6 and E7 cause mutations in the Retinoblastoma $(\mathrm{Rb})$ and p53 gene, thereby leading to uncontrolled proliferation and reduced apoptosis. In the early days of HPV infection, because viral infected cells are undergoing uncontrolled proliferation without undergoing apoptosis, these abnormal cells are not able to fool the immune system of the host. CMI acts successfully against the lesion over a period of 2 - 3 years and the II acts successfully against the virus itself. The most likely player of II in early days of HPV infection is the Interferon (IFN) secreted by the viral-infected cells. IFN slows down viral replication in host cells. This is one way of attacking the virus invading the human body. Another way is by phagocytosis. The three key immune cells involved in the intracellular killing of the virus by phagocytosis are neutrophils, macrophages, and eosinophils [8]. The key players in CMI against the lesion could be the Th9 subset of helper CD4+ T cell, the cytokines IL-2, IFN-gamma, IL-12 and IL-21 [4].

The same explanation holds for the genesis of HSIL as that which holds for the natural regression of LSIL, the difference being that here the immune attack against the virus itself is impaired possibly because of the persistent infection. It is also possible that the HPV infection became persistent because the II failed. Other reasons for HPV infection becoming persistent could be regular exposure to unsafe sex, bad habits like smoking, etc. So, even if on one hand immune system is efficiently attacking lesion, the lesion does not go away because the infection is persistent. The treatment for HSIL, that has been followed, is Loop Electro-surgical Excisional Procedure (LEEP) [9]. The word loop above derives from the "Wire Loop" that the doctor uses to pass electric current in the Transformation Zone (TZ) of the cervix and the lesion so that both are thermally cut to a depth of approximately $8 \mathrm{~mm}$ and a length-breadth of roughly 4 to $5 \mathrm{~mm}$ beyond the lesion.

Notably, the target cells of the HPV-16 and HPV-18 infection, the stem cells of the cervical epithelium are located in the TZ [10]. Hence, it is clear that LEEP clears away the HPV.

If in HSIL, and in some rare cases of LSIL, a certain batch of proliferating viral-infected cells (instead of showing resistance to apoptosis) start undergoing apoptosis at a rate equal to that of proliferation, then these abnormal cells of the cervix are not available

Citation: Pushpam Kumar Sinha. "Pre-cancer Lesions in the Cervix and Cervical Cancer Explained by Fooling of Homeostasis Hypothesis". Acta Scientific Women's Health 3.5 (2021): 10-12. 
to induce immune reaction against themselves. The immune system is blind to these cells, and in the process while it takes time to up-regulate the pentose phosphate pathway these abnormal cells undergo genetic changes to evade the immunity. Thereby, once the pentose phosphate pathway is over-activated, CC sets in. The possible mechanisms of immune evasion in CC could be the secretion of cytokine IL-17 [4], Th17/Treg imbalance [4], down-regulation of HLA-A2 (by HPV-16 E5 protein) [11], etc. Because the apoptotic pathways under the control of $\mathrm{Rb}$ and $\mathrm{p} 53$ proteins have already been (negatively) affected, the increased apoptosis of a certain batch of viral-infected cells in LSIL and HSIL could involve other apoptotic players like, for e.g., PTEN protein [12], miR-17-5p [13], miR-34b [14], etc.

\section{Conclusion}

The known chemicals that slow down pentose phosphate pathway are ethanol-alcohol dehydrogenase and sodium metabisulphite [7]. Hence a possible cure for CC could be to target these chemicals against only the cancer cells. Monoclonal antibodies carrying these toxic chemicals (toxic against tumor) can be used to selectively target only the tumor cells (recognized by the surface antigens).

\section{Bibliography}

1. S Joshi and J M Perez. "Screening for Carcinoma Cervix". Acta Scientific Women's Health 3.3 (2021): 61-65.

2. T Sasagawa., et al. "Immune responses against human papillomavirus (HPV) infection and evasion of host defense in cervical cancer". Journal of Infection and Chemotherapy 18 (2012): 807-815.

3. MR Corces-Zimmerman and R Majeti. "Pre-leukemic evolution of hematopoietic stem cells: The importance of early mutations in leukemogenesis". Leukemia 28 (2014): 2276-2282.

4. L Guo and K Hua. "Cervical Cancer: Emerging Immune Landscape and Treatment". Oncology Targets and Therapy 13 (2020): 8037-8047.

5. P K Sinha. "The common genesis of all cancers". Journal of Carcinogenesis and Mutagenesis 10 (2019): 342.

6. K Wang and D Sun. "Cancer stem cells of hepatocellular carcinoma”. Oncotarget 9.33 (2018): 23306-23314.
7. P Beaconsfield and HW Reading. "Pathways of glucose metabolism and nucleic acid synthesis". Nature 202 (1964): 464-466.

8. S K Mohanty and K Sai Leela. Textbook of Immunology. Jaypee Brothers Medical Publishers (P) Ltd. $2^{\text {nd }}$ edition (2014).

9. Sadan., et al. "Treatment of high-grade squamous intraepithelial lesions: A "see and treat" versus a three-step approach". European Journal of Obstetrics and Gynecology and Reproductive Biology 131 (2007): 73-75.

10. J Organista-Nava., et al. "Cervical cancer stem cell-associated genes: Prognostic implications in cervical cancer (Review)". Oncology Letters 18 (2019): 7-14.

11. MS Campo., et al. "HPV-16 E5 down-regulates expression of surface HLA class I and reduces recognition by CD8 T cells". Virology 407 (2010): 137-142.

12. Li-na Peng., et al. "Effect of miR-301a/PTEN pathway on the proliferation and apoptosis of cervical cancer". Innate Immunity 25.4 (2019): 217-223.

13. Q Wei., et al. "MiR-17-5p Targets TP53INP1 and Regulates Cell Proliferation and Apoptosis of Cervical Cancer Cells". IUBMB Life (2012).

14. Z Cao., et al. "MiR-34b regulates cervical cancer cell proliferation and apoptosis". Artificial Cells, Nanomedicine, and Biotechnology 47.1 (2019): 2042-2047.

\section{Assets from publication with us}

- Prompt Acknowledgement after receiving the article

- Thorough Double blinded peer review

- Rapid Publication

- Issue of Publication Certificate

- High visibility of your Published work

Website: www.actascientific.com/

Submit Article: www.actascientific.com/submission.php

Email us: editor@actascientific.com

Contact us: +919182824667 